\title{
Mannosylglycerate is essential for osmotic adjustment in Thermus thermophilus strains HB27 and RQ-1
}

\author{
Susana Alarico $\cdot$ Nuno Empadinhas $\cdot$ Ana Mingote $\cdot$ \\ Catarina Simões $\cdot$ Maria S. Santos $\cdot$ Milton S. da Costa
}

Received: 25 January 2007/ Accepted: 31 July 2007/Published online: 29 August 2007

(C) Springer 2007

\begin{abstract}
We disrupted the mpgS encoding mannosyl3-phosphoglycerate synthase (MpgS) of Thermus thermophilus strains $\mathrm{HB} 27$ and RQ-1, by homologous recombination, to assess the role of the compatible solute mannosylglycerate (MG) in osmoadaptation of the mutants, to examine their ability to grow in NaCl-containing medium and to identify the intracellular organic solutes. Strain HB27 accumulated only MG when grown in defined medium containing $2 \% \mathrm{NaCl}$; mutant HB27M9 did not grow in the same medium containing more than $1 \%$ $\mathrm{NaCl}$. When trehalose or MG was added, the mutant was able to grow up to $2 \%$ of $\mathrm{NaCl}$ and accumulated trehalose or MG, respectively, plus amino acids. T. thermophilus RQ-1 grew in medium containing up to $5 \% \mathrm{NaCl}$, accumulated trehalose and lower amounts of MG. Mutant RQ$1 \mathrm{M} 1$ lost the ability to grow in medium containing more than $3 \% \mathrm{NaCl}$ and accumulated trehalose and moderate levels of amino acids. Exogenous MG did not improve the ability of the organism to grow above $3 \% \mathrm{NaCl}$, but caused a decrease in the levels of amino acids. Our results show
\end{abstract}

Communicated by A. Driessen.

S. Alarico · N. Empadinhas · C. Simões · M. S. Santos Centro de Neurociências e Biologia Celular e

Departamento de Zoologia, Universidade de Coimbra, 3004-517 Coimbra, Portugal

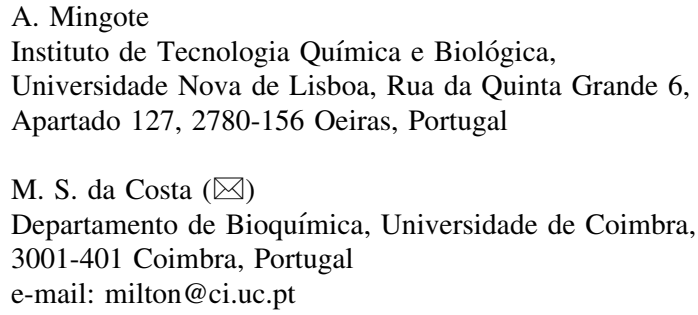

that $\mathrm{MG}$ serves as a compatible solute primarily during osmoadaptation at low levels of $\mathrm{NaCl}$ while trehalose is primarily involved in osmoadaptation during growth at higher $\mathrm{NaCl}$ levels.

Keywords Thermus thermophilus - Mutants · Compatible solutes · Mannosylglycerate .

Trehalose $\cdot$ Osmotic adjustment

\section{Introduction}

The vast majority of the strains of the thermophilic bacteria of the genus Thermus have optimum growth temperatures of $70-75^{\circ} \mathrm{C}$, being commonly isolated from continental hydrothermal areas where the levels of sodium are, generally, low and do not grow in media containing more than $1 \% \mathrm{NaCl}$ (da Costa et al. 2001). However, the strains of the species Thermus thermophilus, frequently isolated from marine hot springs, where the levels of sodium can reach as those of seawater, are halotolerant. Many of these strains accumulate primarily trehalose and lesser amounts of mannosylglycerate (MG) in response to osmotic stress (Nunes et al. 1995; da Costa et al. 2001; Alarico et al. 2005).

Trehalose is a very common compatible solute of prokaryotes and eukaryotes, while MG, initially identified in marine red algae, has been frequently encountered in hyper/ thermophilic bacteria and archaea, but has not yet identified in mesophilic members of these two domains (Bouveng et al. 1955; Nunes et al. 1995; Martins et al. 1997; Silva et al. 1999; Elbein et al. 2003; Neves et al. 2005). The synthesis of trehalose in $T$. thermophilus strains proceeds via the trehalose-phosphate synthase/trehalose-6-phosphate phosphatase (TPS/TPP) pathway coded by otsA/otsB genes 
(Silva et al. 2003; Henne et al. 2004; Silva et al. 2005a) and via the trehalose synthase (TreS) encoded by treS that catalyzes the conversion of maltose into trehalose (Koh et al. 1998; Wang et al. 2007). We previously found that the partial deletion of otsA and otsB genes in T. thermophilus strain RQ-1 reduced the ability of the bacterium to grow in a defined medium (TDM) containing $\mathrm{NaCl}$ from $5 \%$ in the wild-type to $3 \%$ in the deletion mutant. Moreover, the addition of trehalose to the growth medium relieved the salt sensitivity of this mutant providing crucial evidence for the importance of trehalose in the osmotic adaptation in T. thermophilus RQ-1 (Silva et al. 2003).

In T. thermophilus HB27 the biosynthesis of MG involves mannosyl-3-phosphoglycerate synthase (MpgS) that converts GDP-mannose and 3-phosphoglycerate into mannosyl-3-phosphoglycerate (MPG), which is subsequently dephosphorylated to MG by a mannosyl-3phosphoglycerate phosphatase (MpgP) (Empadinhas et al. 2003). Most strains of T. thermophilus, such as RQ-1, PRQ-14, T-2, B and Fiji3 A1, utilize the combined accumulation of trehalose and $\mathrm{MG}$ for growth in a saltcontaining medium. These organisms possess the otsA/ otsB/treS gene cluster as well as the $m p g S / m p g P$ genes. Other strains of $T$. thermophilus, namely HB8, HB27, AT62 and GK24, accumulate only MG. Strains HB8, AT-62 and GK24 possess complete otsB/treS genes and a partial otsA gene, while strain HB27 lacks the otsA/otsB/treS gene cluster, does not grow in the defined medium containing more than $2 \% \mathrm{NaCl}$. Strain CC-16, lacking $m p g S / m p g P$, but possessing the trehalose gene cluster, only accumulates small amounts of trehalose and is not able to grow in TDM containing more than $1 \% \mathrm{NaCl}$ (Alarico et al. 2005).

In the present study we constructed mutants defective in the production of $\mathrm{MG}$, by disrupting the $m p g S$ gene via replacement recombination, to evaluate the role of this solute in osmoadaptation of $T$. thermophilus strains RQ-1 and HB27. The data obtained here provide additional evidence that MG synthesis is necessary for growth of the strains in defined medium containing low levels of $\mathrm{NaCl}$. Moreover, our results show that the accumulation of amino acids does not completely replace $\mathrm{MG}$, which has other unidentified roles in osmotic adjustment.

\section{Materials and methods}

Strains, plasmids and culture conditions

Thermus thermophilus strain HB27 (=DSMZ 7039) was obtained from the Deutsche Sammlung von Mikroorganismen und Zellkulturen, Braunschweig, Germany and T. thermophilus strain RQ-1 (=DSMZ 9247) is a laboratory strain (da Costa et al. 2001). The strains were grown at $70^{\circ} \mathrm{C}$ and $\mathrm{pH} 8.2$ in Thermus medium (DSMZ medium 1033, http://www.dsmz.de/media/med01033.htm) containing tryptone $\left(1.0 \mathrm{~g} \mathrm{l}^{-1}\right)$ and yeast extract $\left(1.0 \mathrm{~g} \mathrm{l}^{-1}\right)$ or in a defined medium (TDM) containing basal salts of Degryse medium 162 (Degryse et al. 1978), tryptone $\left(2.0 \mathrm{~g} \mathrm{l}^{-1}\right)$ and a vitamin solution (Silva et al. 2003). Escherichia coli XL1-blue was used as host for cloning vectors and was grown in YT medium (Silva et al. 2003). Ampicillin was added to the medium $\left(100 \mu \mathrm{g} \mathrm{ml}^{-1}\right)$, for plasmid selection. Transformation assays of the strains HB27 and RQ-1 were performed in a rich medium containing tryptone $\left(8.0 \mathrm{~g} \mathrm{l}^{-1}\right)$, yeast extract $\left(4.0 \mathrm{~g} \mathrm{l}^{-1}\right), \mathrm{NaCl}\left(3.0 \mathrm{~g} \mathrm{l}^{-1}\right), \mathrm{MgCl}_{2}\left(0.2 \mathrm{~g} \mathrm{l}^{-1}\right)$, $\mathrm{CaCl}_{2}\left(0.05 \mathrm{~g} \mathrm{l}^{-1}\right)$ and Castenholz salts (http://www.dsmz. de/media/med086.htm) with a final pH of 8.2. Kanamycin was added to agar plates, or, when needed, to liquid media $\left(30 \mu \mathrm{g} \mathrm{ml}^{-1}\right.$ ).

Plasmid pMK18 was used as a source of the kat gene encoding a thermostable resistance to kanamycin (Lasa et al. 1992). Plasmid pGEM-T Easy (Promega) was used to clone the mpgS gene from strain HB27, and for transformation and recombination of the slpA-kat disrupted mpgS gene in strains HB27 and RQ-1.

\section{DNA manipulations}

The isolation of DNA was performed as previously described (Nielsen et al. 1995). PCR amplifications of the $m p g S$ gene $(1,176 \mathrm{bp})$ from strain HB27 were performed with GC-RICH PCR system kit (Roche). The forward primer TT1 (5'-CGCGAATTCATGCGTCTGGAGATTC CC- $\left.3^{\prime}\right)$ and the reverse primer TT2 (5'-GCGAAGCTTT CATGGCACCCGGAAGCG- $3^{\prime}$ ) were designed based on $m p g S$ sequence of strain HB27 (GenBank AY193871). The PCR product was visualized on $1 \%$ agarose gel electrophoresis and purified by band excision (Promega).

Construction and confirmation of T. thermophilus mpgS disruption mutants

The $m p g S$ gene from strains HB27 and RQ-1 which share $100 \%$ identity at the nucleotide level (Alarico et al. 2005) was cloned into pGEM-T Easy Vector to give pGEMmpgs. A 1,217-bp fragment containing the slpA-kat cassette was removed from pMK18 by digestion with BamHI. This fragment was then subcloned in the BglII restriction site (compatible ends for Bam HI) of pGEM-mpgs resulting in a construction containing a disruption of $m p g S$ gene, named pGEM-mp(k)gs. The orientation of kat gene-insert was confirmed by restriction analysis. Competent $E$. coli cells were transformed with this construction as previously described (Sambrook et al. 1989). Cells were grown 
overnight in medium containing $100 \mu \mathrm{g} \mathrm{ml}^{-1}$ of ampicillin at $37^{\circ} \mathrm{C}$ for plasmid isolation (Roche). The positive clones were sequenced at AGOWA GmbH (Berlin, Germany). Plasmid pGEM-mp(k)gs was then used to transform strains HB27 and RQ-1 and disrupt the native $m p g S$ by double crossover, as previously described (Koyama et al. 1986; De Grado et al. 1999). For this purpose, the host strains were grown at $65^{\circ} \mathrm{C}$ in the transformation medium described above, until the turbidity reached $0.4\left(\mathrm{OD}_{610} \mathrm{~nm}\right)$. Aliquots of cell culture $(1 \mathrm{ml})$ were transferred to $50-\mathrm{ml}$ sterile tubes and $20 \mu \mathrm{g}$ of plasmid DNA was added for transformation. Cells were directly plated onto Thermus agar plates with kanamycin and incubated at 55,60 and $65^{\circ} \mathrm{C}$, after $1.5 \mathrm{~h}$ of incubation at $65^{\circ} \mathrm{C}$ under strong aeration. The chromosomal DNA from transformants was isolated as described above and checked for the expected mutation by Southern blot analysis and PCR with primers TT1 and TT2, primers kan1 (5'-GAAGATCTAGTATAACAGAAACCTTAAG GCCCGAC- $3^{\prime}$ ) and kan2 (5'-GAAGATCTCATCTGTG CGGTATTTCACACC-3'), designed based on the slpA-kat cassette of pMK18, and with primers kan2 and TT4 (5'-GCGCTGCAGTCAGGGCCCGCTCCCTCC- $3^{\prime}$ ). The later primer was designed based on the $3^{\prime}$ end of $m p g P$ gene. Southern blot analysis was performed as previously described (Alarico et al. 2005). Purified DNA (5 $\mu \mathrm{g})$ was digested overnight with BamHI/PstI and electrophoresed on $1 \%$ agarose gels. DIG-labeled $\mathrm{mpgS}$ gene from strain HB27 and DIG-labeled kat gene (PCR fragment of about $963 \mathrm{bp}$ from pMK18) were used as probes to distinguish between wild-type and mutants.

The 16S rRNA gene and the $m p g P$ gene of mutants were amplified and sequenced (AGOWA) to confirm that the mutation was introduced in the desired parental strains. The quality of $16 \mathrm{~S}$ rRNA and mpgP gene sequences was checked manually using the BioEdit sequence editor (Hall 1999) and aligned against the representative gene sequences of $T$. thermophilus strains obtained from EMBL, using CLUSTAL X (Thompson et al. 1997).

Growth conditions and phenotypic analysis

The TDM medium was used without additional $\mathrm{NaCl}$ or supplemented with $\mathrm{NaCl}$ to a final concentration of $1-6 \%$ $(\mathrm{w} / \mathrm{v})$. Growth of the organisms in TDM was also performed with the addition of filter-sterilized trehalose (Sigma), MG (Bitop GmbH, Germany) or both at final concentrations of $0.5 \mathrm{mM}$ and $0.75 \mathrm{mM}$, respectively. Inocula for experimental cultures containing $1 \% \mathrm{NaCl}$ were grown in TDM without additional $\mathrm{NaCl}$. Inocula from medium containing $1 \% \mathrm{NaCl}$ were used for experimental cultures containing $2 \% \mathrm{NaCl}$ or higher. All cultures were grown into 300-ml metal-capped Erlenmeyer flasks, containing $100 \mathrm{ml}$ of medium, with an initial turbidity of $0.05\left(\mathrm{OD}_{610}\right)$ and incubated in a reciprocal-water bath shaker $(120 \mathrm{rpm})$ at $70^{\circ} \mathrm{C}$. The culture medium, for growth of mutants, did not contain kanamycin but their ability to grow in the presence and absence of kanamycin was always tested on solid medium during and after cultivation.

Extraction and determination of intracellular organic solutes

The cells of $T$. thermophilus strains and mutants were harvested by centrifugation $\left(7,000 \times \mathrm{g}, 10 \mathrm{~min}, 4^{\circ} \mathrm{C}\right)$ during mid-exponential growth $\left(\mathrm{OD}_{610}=0.3-0.4\right)$. The extraction of solutes, protein determinations and analysis by NMR were performed as previously described (Silva et al. 2003). The amino acid content in cells extracts was analysed in a Gilson-ASTED HPLC system according to the manufacturer's manual and separated on a Spherisorb ODS column (150 mm $\times 4.6 \mathrm{~mm}$ i.d., $5 \mu \mathrm{m}, \mathrm{C} 18$, Rainin Microsorb) at a flow rate of $2.5 \mathrm{ml} / \mathrm{min}$ for $45 \mathrm{~min}$, using a ternary solvent system as described by Santos et al. (1996). Amino acids were detected as fluorescent derivatives after precolumn derivatization with OPA/MCE ( $O$-phthaldialdehyde/2-mercaptoethanol), at excitation and emission wavelengths of 340 and $410 \mathrm{~nm}$, respectively, using a Gilson fluorescent detector, model 121. The amino acid concentrations were determined by following the peak areas of standard solutions.

\section{Results}

Construction, isolation and confirmation of $T$. thermophilus HB27 mpgS mutants

Mutants derived from the parental T. thermophilus HB27 and RQ-1 strains were formed by homologous recombination using the pGEM-mp(k)gs construct. The coding region of the $m p g S$ gene was only interrupted and no region or nucleotide was removed by the insertion of kat gene as shown by sequencing of the construct. Several HB27 and RQ-1 kanamycin-resistant colonies were clearly seen after 2 days of incubation on Thermus medium agar plates with kanamycin. All HB27 and RQ-1 transformants grew on Thermus medium with kanamycin at $70^{\circ} \mathrm{C}$ and were resistant to the antibiotic after successive transfers in media without kanamycin.

The DNA isolated from all HB27 and RQ-1 transformants was further checked for the required mutation. Mutants from whom a 1,176 bp fragment was amplified with primers TT1/TT2 indicated that no recombination event occurred and were considered negative. Six mutants 
(three mutants from each parental strain) contained a 2,392 bp fragment amplified with primers TT1/TT2 corresponding to the $m p g S$ gene with the inserted $\operatorname{slpA-kat}$ cassette and a 2,006 bp fragment amplified with primers Kan2/TT4 confirming the correct insertion on the genome of strains (Fig. 1a). A fragment of $963 \mathrm{bp}$ was also amplified with primers kan1/kan2 confirming that the kanamycin gene successfully recombined into the genome of wild-type strains (Fig. 1a).

One mutant of each wild-type strain, designated HB27M9 and RQ-1M1, was selected for further confirmation of insertion of the kat gene by Southern blot analysis. The digestion of the DNA from the wild-type strains HB27 and RQ-1 with BamHI/PstI, followed by

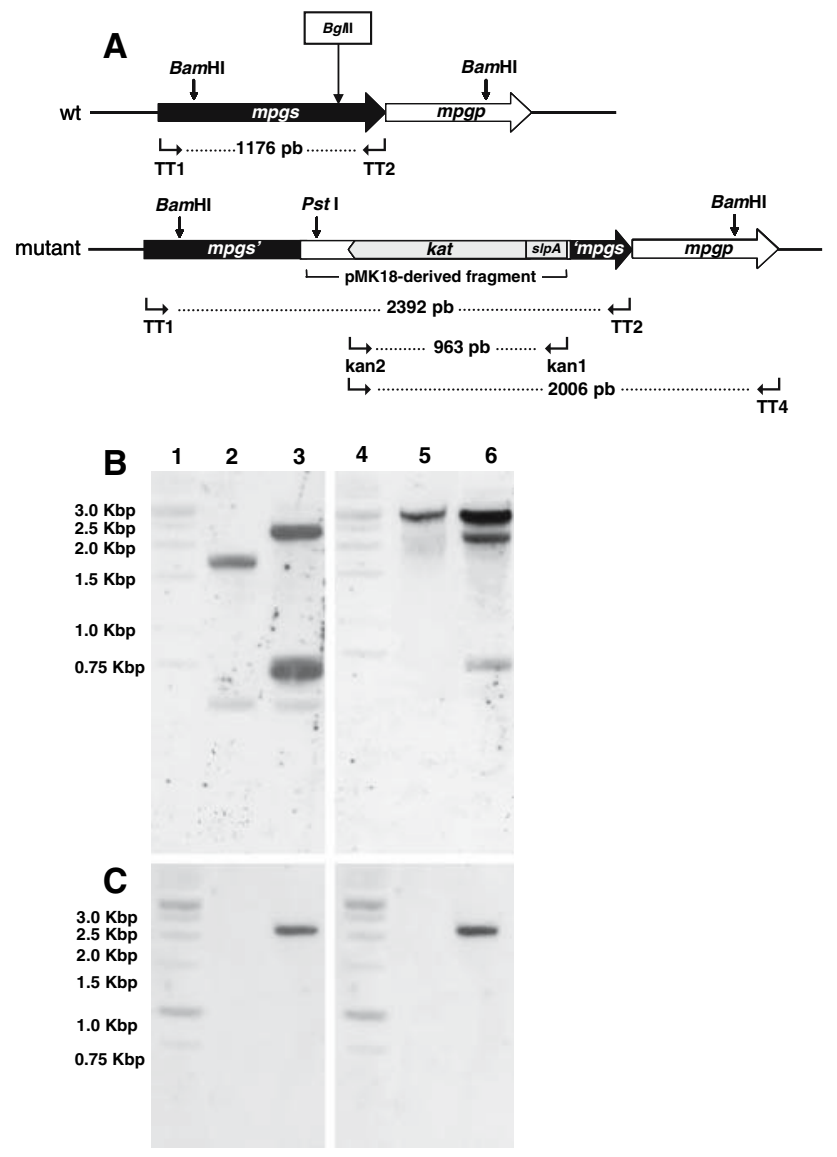

Fig. 1 Southern blot analysis confirming the interruption of $m p g S$ gene, using DNA from strains HB27 and RQ-1 and from the corresponding kanamycin-resistant mutants (HB27M9 and RQ-1M1) digested with BamHI/PstI. a Scheme of the genetic organization of mannosylglycerate-synthesizing genes in the chromosome of the wild-type T. thermophilus (wt) strains HB27 and RQ-1 and in mutants HB27M9 and RQ-1M1 after insertion of a kanamycin resistance cassette, with representation of primers TT1/TT2, kan1/kan2 and kan2/TT4 used for PCR amplifications. b Bands were located by hybridization with an 1,176 bp probe corresponding to the $m p g S$ gene and $\mathbf{c}$ with a 960 bp probe corresponding to the kat gene. Lanes 1 and 4 , molecular weight marker; lane 2, wild-type HB27; lane 3, mutant HB27M9; lane 5, wild-type RQ-1; lane 6, mutant RQ-1M1 hybridization with the $m p g S$ probe, produced two bands (Fig. 1b, lanes 2 and 5). The sizes of the two bands (533 and 1,614 bp) from strain HB27 were verified from the genome sequence (Henne et al. 2004). Of the two visible bands from RQ-1, only the size of one band could be estimated $(1,614 \mathrm{bp})$, since the position of the BamHI restriction site upstream the $m p g S$ gene is not known (Fig. 1b, lane 5). Mutants HB27M9 and RQ-1M1 exhibited three bands by digestion with Bam HI/PstI, since the correct insertion of the fragment containing the slpA-kat cassette led to the introduction of a PstI site (Fig. 1a, b, lanes 3 and 6). The size of the three bands for mutant HB27M9 was estimated to be 533, 690 and 2,141 bp, and for mutant RQ$1 \mathrm{M} 1$ we could only estimate the size of two of the three bands, 690 and 2,141 bp (Fig. 1b, lanes 3 and 6). Moreover, probing with the kat gene indicated its presence only in the mutant strains (Fig. 1c).

Growth of the organisms and accumulation of compatible solutes

Growth rates and the final cell yields of wild-type strains HB27 and RQ-1, as well as mutants HB27M9 and RQ$1 \mathrm{M} 1$, were similar in TDM without additional salt. Trehalose and MG were not detected by NMR, but the total amount of amino acids detected by HPLC was about $1.6 \mathrm{nmol} / \mathrm{mg}$ protein (glutamate, serine and valine being the major amino acids), the values of which cannot be seen in the graphs (Figs. 2, 3). The growth rate of strains HB27 and RQ-1 in TDM decreased as the concentration of $\mathrm{NaCl}$ increased until growth ceased in medium containing 3 and $6 \%$ of $\mathrm{NaCl}$, respectively, corroborating previous results (Silva et al. 2003; Alarico et al 2005). Strain HB27 accumulated primarily $\mathrm{MG}$, which increased in concentration during growth in TDM containing 1 and $2 \% \mathrm{NaCl}$, but did not accumulate trehalose or appreciable levels of glutamate (Fig. 2a), which was the most abundant of the amino acids (70-100\%) detected under all experimental conditions, the remainder being made up of serine, arginine, alanine and valine. Strain RQ-1 accumulated primarily trehalose, followed by lesser amounts of MG. Low amounts of amino acids (glutamate, serine, alanine and valine) were detected in cells derived from TDM containing between 1 and $3 \%$ of $\mathrm{NaCl}$, but these decreased to very low levels in the medium containing 4 and $5 \% \mathrm{NaCl}$ (12 and $3 \mathrm{nmol} / \mathrm{mg}$ protein, respectively) (Fig. 3a).

Mutants HB27M9 and RQ-1M1 were less halotolerant than their respective parental strains. Mutant HB27M9 grew only in TDM containing $1 \% \mathrm{NaCl}$, while mutant RQ$1 \mathrm{M} 1$ grew in the medium supplemented with $3 \% \mathrm{NaCl}$. Only very low levels of amino acids (17 nmol/mg protein) were detected in mutant HB27M9 during growth in TDM 
Fig. 2 Effect of the $\mathrm{NaCl}$ concentration on the growth rate and accumulation of solutes by T. thermophilus strain HB7 and mutant HB27M9 in TDM with $0-3 \% \mathrm{NaCl}$ : in the absence of exogenous solutes (a and $\mathbf{c}$ ) or in presence of trehalose (b and d), mannosylglycerate (e) or simultaneously trehalose and mannosylglycerate (f). Bars represent intracellular concentrations of mannosylglycerate (filled square), trehalose (open square), glutamate (light shaded square) and total of amino acids (striped square)
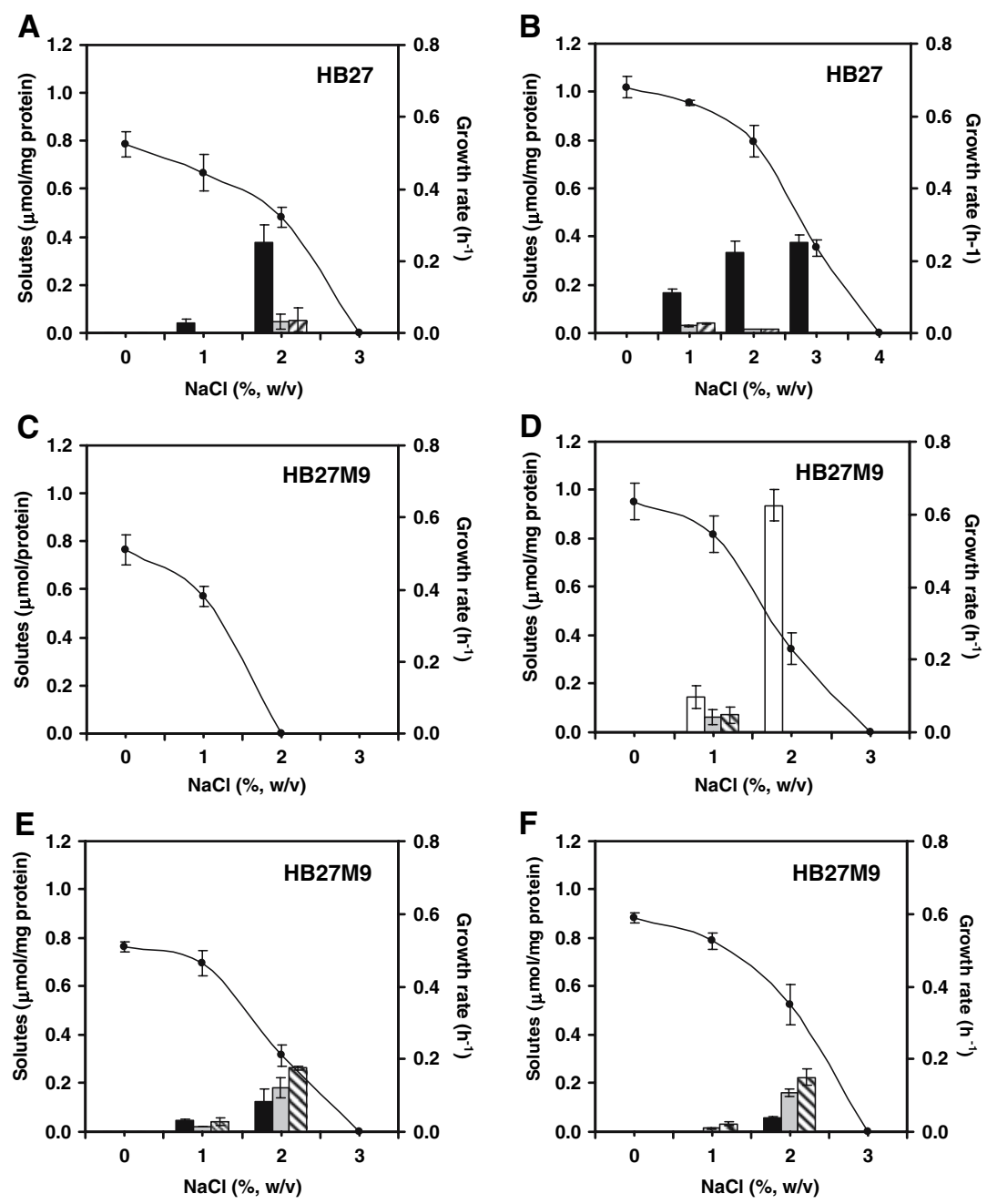

Fig. 3 Effect of the $\mathrm{NaCl}$ concentration on the growth rate and accumulation of solutes by T. thermophilus strain RQ-1 and mutant RQ-1M1 in TDM with $0-6 \% \mathrm{NaCl}$; in the absence of exogenous solutes (a and $\mathbf{b}$ ) or in presence of

mannosylglycerate (c). Bars represent intracellular concentrations of mannosylglycerate (filled square), trehalose (open square), glutamate (light shaded square), total of amino acids (striped square)

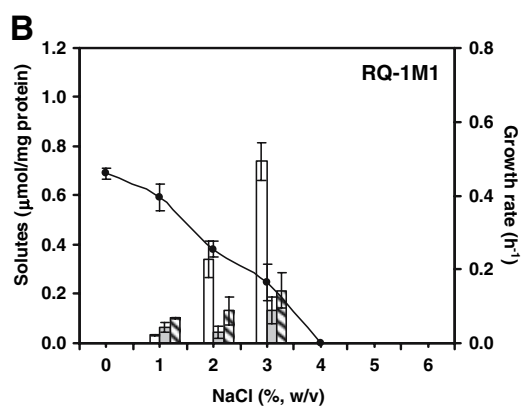

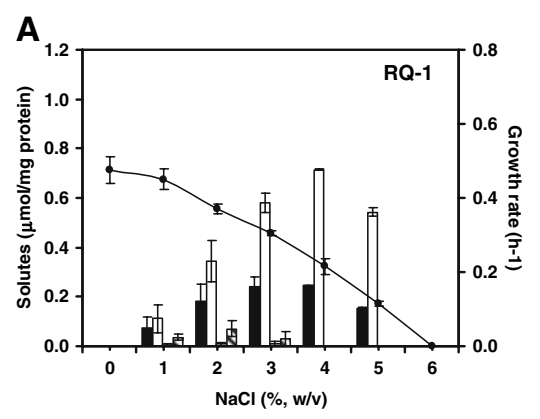

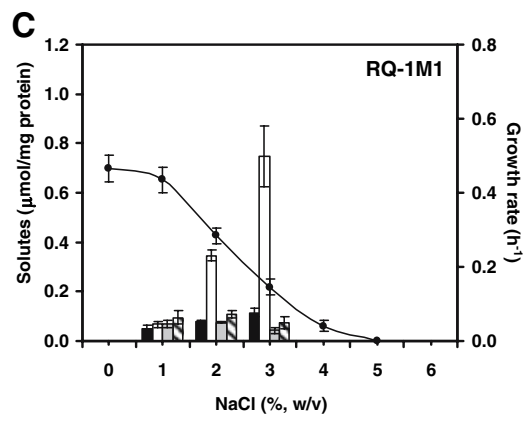


(Fig. 2c). The RQ-1M1 mutant, on the other hand, accumulated larger amounts of trehalose than the wild-type strain in TDM containing 2 and $3 \%$ of $\mathrm{NaCl}$ and, as expected, MG was not detected. This mutant also accumulated appreciable amounts of amino acids that attained $0.21 \mu \mathrm{mol} / \mathrm{mg}$ protein in medium containing $3 \% \mathrm{NaCl}$ (Fig. 3b).

The addition of trehalose to TDM led to an increase in the ability of strain HB27 and mutant HB27M9 to grow in medium containing higher levels of $\mathrm{NaCl}$. The wild-type strain was able to grow in medium containing $3 \% \mathrm{NaCl}$ and the mutant in medium containing $2 \% \mathrm{NaCl}$ (Fig. 2b, d). The wild-type HB27 did not accumulate trehalose, but the levels of MG increased progressively in TDM containing between 1 and $3 \% \mathrm{NaCl}$, while the levels of amino acids remained vestigial (Fig. 2b). Under these conditions, mutant HB27M9 accumulated trehalose in medium containing 1 and $2 \% \mathrm{NaCl}$ and glutamate, which decreased to almost undetectable levels in $2 \% \mathrm{NaCl}$-containing medium (Fig. 2d). The addition of $\mathrm{MG}$ to the medium resulted in the growth of mutant HB27M9 in TDM containing 2\% $\mathrm{NaCl}$ and led to the accumulation of high levels of glutamate $(0.18 \mu \mathrm{mol} / \mathrm{mg}$ protein $)$ that exceeded the levels of MG $(0.13 \mu \mathrm{mol} / \mathrm{mg}$ protein) taken up from the medium (Fig. 2e). The simultaneous addition of $\mathrm{MG}$ and trehalose to TDM containing $2 \% \mathrm{NaCl}$ led to an increase in the growth rate of mutant $\mathrm{HB} 27 \mathrm{M} 9$ by about $60 \%$, compared to the growth rates when $\mathrm{MG}$ and trehalose were added separately, but did not extended growth beyond this level of salt. Under these conditions the organism accumulated only small amounts of $\mathrm{MG}(0.06 \mu \mathrm{mol} / \mathrm{mg}$ protein $)$ and higher amounts of glutamate $(0.16 \mu \mathrm{mol} / \mathrm{mg}$ protein $)$ and amino acids $(0.23 \mu \mathrm{mol} / \mathrm{mg}$ protein $)$ in medium containing $2 \% \mathrm{NaCl}$, but trehalose did not accumulate (Fig. 2f).

The addition of MG did not extend the ability of mutant RQ-1M1 to grow in TDM containing levels of $\mathrm{NaCl}$ higher than $3 \%$, although MG was taken up by the cells and low levels accumulated under salt stress. Under these conditions trehalose continued to be the dominant compatible solute in mutant RQ-1M1, followed by lower amounts of MG and amino acids (Fig. 3c).

\section{Discussion}

In this study we investigated the role of $\mathrm{MG}$ in the osmotic adjustment of $T$. thermophilus strains HB27 and RQ-1 by disrupting the $m p g S$ gene, leading to mutants unable to synthesize $\mathrm{MG}$ and to grow in media with $\mathrm{NaCl}$ above 1 or $3 \%$, respectively. We had previously examined the role of trehalose in osmotic adjustment of strain RQ-1 and the results indicated that this disaccharide was necessary for the organism to grow in TDM containing $\mathrm{NaCl}$ above about 3\%, even though the organism also accumulated MG (Silva et al. 2003). These results led us to speculate that MG was necessary for osmotic adjustment of strain RQ-1 to low levels of $\mathrm{NaCl}$, where it played a similar role to that of glutamate in other organisms. Glutamate is a common compatible solute that appears to serve as a counterion for potassium during osmotic adjustment to low levels of $\mathrm{NaCl}$, being replaced in many organisms by neutral or zwitterionic compatible solutes as the level of $\mathrm{NaCl}$ increases (da Costa et al. 1998; Santos and da Costa 2002).

Strains HB27 and RQ-1 possess different strategies for growth in medium containing $\mathrm{NaCl}$. Strain RQ-1 accumulates primarily trehalose and lower levels of $\mathrm{MG}$, and is able to grow in media containing up to about $5 \% \mathrm{NaCl}$ (Nunes et al. 1995; da Costa et al. 2001; Silva et al. 2003; Alarico et al. 2005). Moreover, the trehalose disruption mutant accumulated trehalose when it was added to medium containing $\mathrm{NaCl}$ and re-established growth with high levels of $\mathrm{NaCl}$ (Silva et al. 2003). The mpgS disruption prevented mutant RQ-1M1 from growing in TDM containing more than $3 \% \mathrm{NaCl}$. This mutant was able to accumulate trehalose, like the wild-type, but not MG during the low level salt stress. Unexpectedly, we found that the mutant accumulated large amounts of glutamate (among other minor amino acids), which were found in only trace levels in the wild-type. These results, along with those showing that the slight accumulation of exogenous MG led to a decrease in the accumulation of amino acids by mutant RQ-1M1, argue for a role of this negativelycharged compatible solute, possibly as a counterion for potassium, during low level osmotic adjustment. However, increased glutamate could not completely replace $\mathrm{MG}$, since this mutant, despite the accumulation of trehalose and glutamate, was not able to grow in the medium containing more than $3 \% \mathrm{NaCl}$, suggesting that $\mathrm{MG}$ also serves an unknown role in media containing higher levels of salt. As reported previously, MG may also protect against heat inactivation of proteins at supraoptimum growth temperatures, although in vivo evidence is still lacking (Borges et al. 2002).

Strain HB27 does not grow in any medium containing more than $2-3 \% \mathrm{NaCl}$. The organism accumulates $\mathrm{MG}$, but does not accumulate trehalose during osmotic adjustment even when trehalose is supplied in the medium. However, previous results show that trehalose serves as a carbon and energy source for these organisms including strains HB27 and RQ-1 (da Costa et al. 2001; Silva et al. 2005a). The $m p g S$ inactivation left mutant HB27M9 without the ability to grow in TDM containing more than $1 \% \mathrm{NaCl}$, like the strains of the other species of Thermus, which also lack genes for the synthesis of trehalose and MG (da Costa et al. 2001; Alarico et al. 2005). This result clearly shows that trehalose and MG together are responsible for the osmotic 
adjustment of the strains of the T. thermophilus (Alarico et al. 2005). This mutant also accumulated high concentrations of glutamate in $2 \% \mathrm{NaCl}$ medium supplemented with exogenous MG, or with exogenous MG and trehalose arguing for a role of MG in low level osmodaptation.

The addition of exogenous trehalose allowed the wildtype $\mathrm{HB} 27$ to grow in medium containing $3 \% \mathrm{NaCl}$, which it could not in the medium without trehalose, but the effect was due to the accumulation of slightly higher levels of MG and not trehalose. Exogenous trehalose led, unexpectedly, to the accumulation of very high levels of this solute by mutant HB27M9 and the ability to grow in $2 \%$ $\mathrm{NaCl}$-containing medium. The accumulation of the disaccharide had never been observed before in strain HB27, despite the presence of a high-affinity maltose/trehalose ABC transporter (Silva et al. 2005b). This result indicated that the organism accumulated a very large amount of trehalose to counterbalance the lack of $\mathrm{MG}$, although it could not lead to osmotic adjustment beyond $2 \% \mathrm{NaCl}$. It is also difficult to explain this result without postulating that the gene or genes involved in the catabolism of trehalose are repressed in the presence of $\mathrm{NaCl}$ and that this repression is somehow related to the presence/absence of intracellular MG. A gene coding for a $\alpha$-glucosidase that hydrolyzes trehalose has been detected in strain HB27 which lacks genes for a classical trehalase (EC 3.2.1.28). This enzyme may be responsible for the utilization of trehalose by strain HB27 and the control of the synthesis of this enzyme is now being examined (Alarico et al. unpublished results). The addition of MG to the medium resulted in the accumulation of low levels of MG and amino acids, indicating a similar regulation of compatible solute accumulation in both mutants. The results obtained from mutant HB27M9 grown in medium with $2 \% \mathrm{NaCl}$, containing both trehalose and $\mathrm{MG}$, again defy a simple explanation since, under these conditions, the primary compatible solutes were amino acids followed by lesser amounts of MG but trehalose was not detected indicating that the accumulation of this solute does not occur when the organism accumulates MG.

The behavior of the mpgS disruption mutants in medium containing $\mathrm{NaCl}$ further supports the notion that MG serves as a compatible solute in T. thermophilus strains HB27 and RQ-1. These results complement previous ones showing that trehalose is primarily involved in osmotic adaptation in medium with $\mathrm{NaCl}$ above about $3 \%$, while $\mathrm{MG}$ is primarily involved in osmotic adaptation in media containing lower levels of $\mathrm{NaCl}$ and that glutamate can, to some extent, replace this compatible solute.

Acknowledgments This work was supported by Fundação para a Ciência e a Tecnologia (FCT), Portugal and FEDER, project POCI/ BIA-MIC/56511/2004. S. Alarico, N. Empadinhas and A. Mingote acknowledge scholarships from FCT (SFRH/BD/14140/2003, SFRH/ BPD/14828/2003 and Project A004/2005 Action V.5.1, respectively). We wish to thank Zélia Silva for advice on the strategies for mutant's construction.

\section{References}

Alarico S, Empadinhas N, Simões C, Silva Z, Henne A, Mingote A, Santos H, da Costa MS (2005) Distribution of genes for synthesis of trehalose and mannosylglycerate in Thermus spp. and direct correlation of these genes with halotolerance. Appl Environ Microbiol 71:2460-2466

Borges N, Ramos A, Raven ND, Sharp RJ, Santos H (2002) Comparative study of the thermostabilizing properties of mannosylglycerate and other compatible solutes on model enzymes. Extremophiles 6:209-216

Bouveng H, Lindberg B, Wickberg B (1955) Low-molecular carbohydrates in algae. Structure of the glyceric acid mannoside from red algae. Acta Chem Scand 9:807-809

da Costa MS, Santos H, Gallinski EA (1998) An overview of the role and diversity of compatible solutes in bacteria and archaea. Adv Biochem Eng Biotechnol 61:118-153

da Costa MS, Nobre MF, Rainey FA (2001) The genus Thermus. In: Boone DR, Castenholtz RW, Garrity GM (eds) Bergeýs manual of systematic bacteriology, vol 1, 2nd edn. Springer, Berlin, pp 404-414

De Grado M, Castán P, Berenguer J (1999) A high-transformationefficiency cloning vector for Thermus thermophilus. Plasmid 42:241-245

Degryse E, Glansdorff N, Pierard A (1978) A comparative analysis of extreme thermophilic bacteria belonging to the genus Thermus. Arch Microbiol 117:189-196

Elbein AD, Pan YT, Pastuszak I, Carroll D (2003) New insights on trehalose: a multifunctional molecule. Glycobiology 13:17-27

Empadinhas N, Albuquerque L, Henne A, Santos H, da Costa MS (2003) The bacterium Thermus thermophilus, like hyperthermophilic archaea, uses a two-step pathway for the synthesis of mannosylglycerate. Appl Environ Microbiol 69:3272-3279

Hall TA (1999) BioEdit: a user-friendly biological sequence alignment editor and analysis program for Windows 95/98/NT. Nucleic Acids Symp Ser 41:95-98

Henne A, Brüggemann H, Raasch C, Wiezer A, Hartsch T, Liesegang H, Johann A, Lienard T, Gohl O, Martinez-Arias R, Jacobi C, Starkuviene V, Schlenczeck S, Denker S, Huber R, Klenk H-P, Kramer W, Merkl R, Gottschalk G, Fritz H-J (2004) The genome sequence of the extreme thermophile Thermus thermophilus. Nat Biotechnol 22:547-553

Koh S, Shin H-J, Kim JS, Lee D-S, Lee SY (1998) Trehalose synthesis from maltose by a thermostable trehalose synthase from Thermus caldophilus. Biotechnol Lett 20:757-761

Koyama Y, Oshino T, Tomizuka N, Furukawa K (1986) Genetic transformation of the extreme thermophile Thermus thermophilus and other Thermus spp. J Bacteriol 166:338-340

Lasa I, Castón JR, Fernandez-Herrero LA, Pedro MA, Berenguer J (1992) Insertional mutagenesis in the extreme thermophilic eubacteria Thermus thermophilus HB8. Mol Microbiol 11:15551564

Martins LO, Huber R, Huber H, Stetter KO, da Costa MS, Santos H (1997) Organic solutes in hyperthermophilic Archaea. Appl Environ Microbiol 63:896-902

Neves C, da Costa MS, Santos H (2005) Osmoadaptation and thermoadaptation in the three genera of the order thermococcales: compatible solutes of the hyperthermophile Palaeococcus ferrophilus. Appl Environ Microbiol 71:8091-8098 
Nielsen P, Fritze D, Priest FG (1995) Phenetic diversity of alkaliphilic Bacillus strains: proposal for nine new species. Microbiology 141:1745-1761

Nunes OC, Manaia CM, da Costa MS, Santos H (1995) Compatible solutes in the thermophilic bacteria Rhodothermus marinus and "Thermus thermophilus". Appl Environ Microbiol 61:2351-2357

Santos H, da Costa MS (2002) Compatible solutes of organisms that live in hot saline environments. Environ Microbiol 4:501-509

Santos MS, Moreno AJ, Carvalho AP (1996) Relationships between ATP depletion, membrane potential, and the release of neurotransmitters in rat nerve terminals. An in vitro study under conditions that mimic anoxia, hypoglycemia, and ischemia. Stroke 27:941-950

Sambrook J, Fritsch EF, Maniatis T (1989) Molecular cloning: a laboratory manual, 2nd edn. Cold Spring Harbor Laboratory, Cold Spring Harbor, New York

Silva Z, Borges N, Martins LO, Wait R, da Costa MS, Santos H (1999) Combined effect of the growth temperature and the salinity of the medium on the accumulation of compatible solutes by Rhodothermus marinus and Rhodothermus obamensis. Extremophiles 3:163-172
Silva Z, Alarico S, Nobre A, Horlacher R, Marugg J, Boos W, Mingote A, da Costa MS (2003) Osmotic adaptation of Thermus thermophilus RQ-1: a lesson from a mutant deficient in the synthesis of trehalose. J Bacteriol 185:5943-5952

Silva Z, Alarico S, da Costa MS (2005a) Trehalose biosynthesis in Thermus thermophilus RQ-1: biochemical properties of the trehalose-6-phosphate synthase and trehalose-6-phoshate phosphatase. Extremophiles 9:29-36

Silva Z, Sampaio MM, Henne A, Böhm A, Gutzat R, Boos W, da Costa MS, Santos H (2005b) The high-affinity maltose/trehalose $\mathrm{ABC}$ transporter in the extremely thermophilic bacterium Thermus thermophilus HB27 also recognizes sucrose and palatinose. J Bacteriol 187:1210-1218

Thompson JD, Gibson TJ, Plewniak F, Jeanmougin F, Higgins DG (1997) The ClustalX windows interface: flexible strategies for multiple sequence alignment aided by quality analysis tools. Nucl Acids Res 24:4876-4882

Wang J-H, Tsai M-Y, Chen J-J, Lee G-C, Shaw J-F (2007) Role of the C-terminal domain of Thermus thermophilus trehalose synthase in the thermophilicity, thermostability, and efficient production of trehalose. J Agric Food Chem 55:3435-3443 